\title{
Morita equivalence bimodules for Wick type star products
}

\author{
Nikolai Neumaier, Stefan Waldmann ${ }^{\S}$ \\ Fakultät für Physik \\ Albert-Ludwigs-Universität Freiburg \\ Hermann Herder Straße 3 \\ D 79104 Freiburg \\ Germany
}

July 2002

FR-THEP 2002/09

\begin{abstract}
In this paper, the notion of star products with separation of variables on a Kähler manifold is extended to bimodule deformations of (anti-) holomorphic vector bundles over a Kähler manifold. Here the Fedosov construction is appropriately adapted using the geometric data of a connection in the vector bundle. Moreover, the relation between the star products of Wick and anti Wick type is clarified by constructing a canonical Morita equivalence bimodule as bimodule deformation of the canonical line bundle over the Kähler manifold.
\end{abstract}

Mathematics Subject Classification (2000): 53D55

Keywords: Deformation quantization, Fedosov star products, Kähler manifolds, Morita equivalence, Separation of variables.

\section{Introduction}

Deformation quantization as introduced in [1] is a well-established and successful way of understanding the transition from classical physics to quantum physics as a deformation of the algebraic structures, see [15, 18, 20, 36] for recent reviews. The classical observable algebra is modelled by the complex-valued smooth functions $C^{\infty}(M)$ on the phase space $M$, which is a Poisson manifold. In particular, $C^{\infty}(M)$ is a Poisson algebra. The deformation is done by means of a star product $\star$ which is an associative $\mathbb{C}[[\lambda]]$-bilinear multiplication for $C^{\infty}(M)[[\lambda]]$ such that in zeroth order of the deformation parameter $\lambda$ it coincides with the pointwise product and in first order of $\lambda$ the $\star$-commutator equals i times the Poisson bracket. In case of convergence $\lambda$ corresponds to Planck's constant $\hbar$.

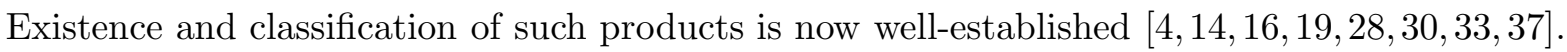

In this article we shall consider a particular situation, namely when $M$ is a Kähler manifold and the Poisson structure is induced by its Kähler symplectic form. Deformation quantization of Kähler manifolds has a long history dating back to the work of Berezin [2,3]. The relation between BerezinToeplitz quantization and star products has been explored by various authors [10 13, 25, 26, 29, 34].

\footnotetext{
*Nikolai.Neumaier@physik.uni-freiburg.de

§Stefan.Waldmann@physik.uni-freiburg.de
} 
Since on a Kähler manifold one has a compatible complex structure it is meaningful to speak of bidifferential operators (and hence of star products) which differentiate one function in holomorphic directions and the other in anti-holomorphic directions only. This property of a star product was known for various examples and led Karabegov to the notion of star products with separation of variables. In [22, 23] he proved existence and gave a classification of such star products. In an alternative approach, Fedosov's construction was adapted to the Kähler situation and used to show the existence of such star products as well [6], see also [32] for a classification in this context and [24] for a comparison of both approaches.

It turns out that on a Kähler manifold one has (at least) three canonically given star products: the Weyl ordered Fedosov star product $\star_{\text {Weyl }}$ as well as the Wick and anti Wick star product $\star_{\text {Wick }}$ and $\star_{\overline{\text { Wick }}}$, obtained by the above mentioned modified Fedosov construction, all using the Kähler connection as starting point. Moreover, the characteristic classes of these three star products are explicitly known to be

$$
c\left(\star_{\text {Weyl }}\right)=\frac{1}{\mathrm{i} \lambda}[\omega], \quad c\left(\star_{\text {Wick }}\right)=\frac{1}{\mathrm{i} \lambda}[\omega]-\mathrm{i} \pi c_{1}\left(L_{\text {can }}\right) \quad c\left(\star_{\overline{\text { Wick }}}\right)=\frac{1}{\mathrm{i} \lambda}[\omega]+\mathrm{i} \pi c_{1}\left(L_{\text {can }}\right),
$$

where $L_{\text {can }}=\bigwedge^{(n, 0)} T^{*} M$ is the canonical line bundle of $M$, see e.g. 21, 26, 32].

In [8] the notion of deformation quantization of vector bundles was introduced: If $E \rightarrow M$ is a (complex) vector bundle and $\star$ is a star product for $M$ then a deformation quantization of $E$ is a deformed right module structure $\bullet$ for $\Gamma^{\infty}(E)[[\lambda]]$ with respect to $\star$. It was shown that such a $\bullet$ always exists and is unique up to equivalence. Moreover, $\bullet$ also induces an associative formal deformation $\star^{\prime}$ of $\Gamma^{\infty}(\operatorname{End}(E))[[\lambda]]$ together with a corresponding left module structure $\bullet^{\prime}$ such that $\Gamma^{\infty}(E)$ is deformed into a $\star^{\prime}-\star$ bimodule via $\bullet^{\prime}$ and $\bullet$. Finally, the bimodule gives a Morita equivalence bimodule for the deformed algebras $\left(\Gamma^{\infty}(\operatorname{End}(E))[[\lambda]], \star^{\prime}\right)$ and $\left(C^{\infty}(M)[[\lambda]], \star\right)$. In case of a line bundle $E=L$ one obtains a star product $\star^{\prime}$ for $C^{\infty}(M)=\Gamma^{\infty}(\operatorname{End}(L))$ and finally arrives at the classification of star products up to Morita equivalence: In the symplectic case two star products $\star^{\prime}$ and $\star$ are Morita equivalent if and only if there is a symplectic diffeomorphism $\psi$ of $M$ such that $\psi^{*} c\left(\star^{\prime}\right)-c(\star) \in 2 \pi \mathrm{iH}_{\text {deRham }}(M, \mathbb{Z})$ is an integral de Rham class, see 9 .

The aim of this work is two-fold: On one hand we shall construct particular bimodule deformations $\bullet^{\prime}, \bullet$ for a complex vector bundle $E$ over $M$ using the ideas of Fedosov's construction as in [35] in order to obtain separation of variables for $\bullet$ ' and $\bullet$ as well, if $E$ is (anti-) holomorphic. On the other hand we give a Fedosov construction of the deformed bimodule structure of $L_{\text {can }}$ which yields a Morita equivalence bimodule for $\star_{\text {Wick }}$ and $\star_{\text {Wick }}$. According to (1) and the general classification theorem in [9] such deformations necessarily exist. So our main emphasize is the canonical and constructive way how it can be obtained.

The paper is organized as follows: In Section 2 we collect some basic and well-known results on the Fedosov construction and adapt them to the Kähler situation. In order to handle the Weyl ordered, the Wick ordered and the anti Wick ordered case simultaneously we introduce a oneparameter family of fibrewise $\kappa$-ordered products. In Section 3 we state the main theorems of the Fedosov construction for the star products and the corresponding bimodule multiplications. Up to here the results are essentially standard. In Section 1 we investigate the case of (anti-) holomorphic vector bundles and show the separation of variables properties of the Wick and anti Wick ordered products. Section 5 contains local expressions for the deformed multiplications which can also be used to characterize them globally. In Section 6 we give a deformed version of a Hermitian fibre metric and investigate its compatibility with the holomorphic structure. Here we also give local expressions. Finally, Section 7 contains the construction of the canonical Morita equivalence bimodule structure on $L_{\text {can }}$ for $\star_{\text {Wick }}$ and $\star_{\text {Wick }}$. In an appendix we have collected some standard results on Kähler geometry in order to explain our notation. 
Conventions: By $C^{\infty}(M)$ we denote the complex-valued smooth functions and similarly $\Gamma^{\infty}\left(T^{*} M\right)$ stands for the complex-valued smooth one-forms etc. Moreover, we use Einstein's summation convention in local expressions. Finally, we do not need the positive definiteness of the Kähler metric. So all results are still valid on semi Kähler manifolds. However, for ease of notation we shall not emphasize this in the text.

Acknowledgements: We would like to thank Martin Schlichenmaier for valuable discussions on topics in Kähler geometry.

\section{The Fedosov construction on Kähler manifolds}

In this section we shall briefly recall the set-up for the Fedosov construction in order to explain our notation where we mainly follow [35]. Details and proofs can be found in Fedosov's book [17]. For the additional structures on a Kähler manifold we refer to [6, 32] as well as to Appendix A.

Given a complex vector bundle $E \rightarrow M$ over a Kähler manifold $M$ we define the following $\mathbb{C}[[\lambda]]$-modules

$$
\begin{gathered}
\mathcal{W}:=\prod_{s=0}^{\infty} \Gamma^{\infty}\left(\bigvee^{s} T^{*} M\right)[[\lambda]], \\
\mathcal{W} \otimes \Lambda^{\bullet}:=\prod_{s=0}^{\infty} \Gamma^{\infty}\left(\bigvee^{s} T^{*} M \otimes \Lambda^{\bullet} T^{*} M\right)[[\lambda]], \\
\mathcal{W} \otimes \Lambda^{\bullet} \otimes \mathcal{E}:=\prod_{s=0}^{\infty} \Gamma^{\infty}\left(\bigvee^{s} T^{*} M \otimes \bigwedge^{\bullet} T^{*} M \otimes E\right)[[\lambda]], \\
\mathcal{W} \otimes \Lambda^{\bullet} \otimes \mathcal{E} n d(\mathcal{E}):=\prod_{s=0}^{\infty} \Gamma^{\infty}\left(\bigvee^{s} T^{*} M \otimes \Lambda^{\bullet} T^{*} M \otimes \operatorname{End}(E)\right)[[\lambda]] .
\end{gathered}
$$

Then $\mathcal{W} \otimes \Lambda^{\bullet}$ becomes a super-commutative associative algebra where the fibrewise product is defined by $(f \otimes \alpha)(g \otimes \beta)=f \vee g \otimes \alpha \wedge \beta$. In particular $\mathcal{W} \subseteq \mathcal{W} \otimes \Lambda^{\bullet}$ is a commutative sub-algebra. Using the fibrewise composition of endomorphisms of $E$ we observe that $\mathcal{W} \otimes \Lambda^{\bullet} \otimes \mathcal{E} n d(\mathcal{E})$ becomes an associative algebra as well, which is no longer super-commutative unless $E=L$ is a line bundle. We can view $\mathcal{W} \otimes \Lambda^{\bullet}$ as sub-algebra of $\mathcal{W} \otimes \Lambda^{\bullet} \otimes \mathcal{E} n d(\mathcal{E})$. Finally, $\mathcal{W} \otimes \Lambda^{\bullet} \otimes \mathcal{E}$ is a bimodule for $\mathcal{W} \otimes \Lambda^{\bullet} \otimes \mathcal{E} n d(\mathcal{E})$ from the left and for $\mathcal{W} \otimes \Lambda^{\bullet}$ from the right. Besides the usual symmetric and anti-symmetric form degree $\operatorname{deg}_{\mathrm{s}}$ and $\operatorname{deg}_{\mathrm{a}}$, we have the $\lambda$-degree and the total degree, which is twice the $\lambda$-degree plus the symmetric degree. One also has the operators $\delta=d x^{i} \wedge i_{s}\left(\partial_{i}\right)$ and $\delta^{*}=d x^{i} \vee i_{a}\left(\partial_{i}\right)$ which satisfy $\delta^{2}=0=\left(\delta^{*}\right)^{2}$. If one defines $\delta^{-1} a=\frac{1}{k+l} \delta^{*} a$ for homogeneous $a$ with symmetric degree $k$ and anti-symmetric degree $l$ such that $k+l \neq 0$ and $\delta^{-1} a=0$ otherwise, then one has the Hodge-de Rham decomposition

$$
\delta \delta^{-1}+\delta^{-1} \delta+\sigma=\mathrm{id},
$$

where $\sigma$ denotes the projection onto the part with symmetric and anti-symmetric degree 0 .

In a next step one needs a symplectic connection $\nabla$ on $M$ and a connection $\nabla^{E}$ for $E$. For $\nabla$ we shall always use the Kähler connection while $\nabla^{E}$ shall be specified later. One has the induced 
connection $\nabla^{\operatorname{End}(E)}=\left[\nabla^{E}, \cdot\right]$ on $\operatorname{End}(E)$ and the connections extend to super-derivations of antisymmetric degree +1

$$
\begin{gathered}
D: \mathcal{W} \otimes \Lambda^{\bullet} \rightarrow \mathcal{W} \otimes \Lambda^{\bullet+1}, \\
D^{E}: \mathcal{W} \otimes \Lambda^{\bullet} \otimes \mathcal{E} \rightarrow \mathcal{W} \otimes \Lambda^{\bullet+1} \otimes \mathcal{E}, \\
D^{\prime}: \mathcal{W} \otimes \Lambda^{\bullet} \otimes \mathcal{E} n d(\mathcal{E}) \rightarrow \mathcal{W} \otimes \Lambda^{\bullet+1} \otimes \mathcal{E} n d(\mathcal{E}) .
\end{gathered}
$$

Then $D^{E}$ is a module derivation along $D$ and $D^{\prime}$, respectively. A simple computation shows that $\delta$ super-commutes with $D^{\prime}, D^{E}$ and $D$.

One observes that the curvature $R^{E}$ of $\nabla^{E}$ is an element in $\mathcal{W} \otimes \Lambda^{2} \otimes \mathcal{E} n d(\mathcal{E})$ with $\operatorname{deg}_{\mathrm{s}} R^{E}=0$. For $R^{E}$ and for the symplectic curvature tensor $R \in \mathcal{W} \otimes \Lambda^{2}$, see (84), we have the Bianchi identities $\delta R=0=\delta R^{E}$ and $D R=0=D^{\prime} R^{E}$.

Now we pass to the deformed fibrewise products and bimodule structures. Originally, Fedosov used the fibrewise Weyl product, but on a Kähler manifold one also has fibrewise analogues of the Wick and anti Wick product, see [6, 24, 32]. First one defines the fibrewise operator

$$
\mathcal{P}=\Lambda^{i j} i_{s}\left(\partial_{i}\right) \otimes i_{s}\left(\partial_{j}\right)
$$

acting on $\mathcal{W} \otimes \mathcal{W}$, where $\Lambda=\frac{1}{2} \Lambda^{i j} \partial_{i} \wedge \partial_{j}$ is the Poisson tensor in local coordinates. Clearly $\mathcal{P}$ is globally defined. As we have a complex structure the operators

$$
P=g^{k \bar{\ell}} i_{s}\left(Z_{k}\right) \otimes i_{s}\left(\bar{Z}_{\ell}\right) \text { and } \bar{P}=g^{k \bar{\ell}} i_{s}\left(\bar{Z}_{\ell}\right) \otimes i_{s}\left(Z_{k}\right)
$$

are globally well-defined as well and one has $\mathcal{P}=\frac{2}{\mathrm{i}}(P-\bar{P})$. Finally, we need the fibrewise Laplace operator

$$
\Delta_{\mathrm{fib}}=g^{k \bar{\ell}} i_{s}\left(Z_{k}\right) i_{s}\left(\bar{Z}_{\ell}\right)
$$

which again is globally well-defined and satisfies the relation

$$
\Delta_{\mathrm{fib}} \circ \mu=\mu \circ\left(\Delta_{\mathrm{fib}} \otimes \mathrm{id}+P+\bar{P}+\mathrm{id} \otimes \Delta_{\mathrm{fib}}\right),
$$

where $\mu: \mathcal{W} \otimes \mathcal{W} \rightarrow \mathcal{W}$ denotes the fibrewise undeformed product of $\mathcal{W}$. We extend all the operators $\mathcal{P}, P, \bar{P}$, and $\Delta_{\text {fib }}$ to $\mathcal{W} \otimes \Lambda^{\bullet}, \mathcal{W} \otimes \Lambda^{\bullet} \otimes \mathcal{E}$, and $\mathcal{W} \otimes \Lambda^{\bullet} \otimes \mathcal{E} n d(\mathcal{E})$. Now the fibrewise Weyl product is defined by

$$
a \circ_{\mathrm{Weyl}} b:=\mu \circ \mathrm{e}^{\frac{\mathrm{i} \lambda}{2} \mathcal{P}} a \otimes b
$$

and clearly gives an associative deformation of $\mu$. For $\kappa \in \mathbb{R}$ we define the fibrewise equivalence transformations

$$
S=\mathrm{e}^{\lambda \Delta_{\mathrm{fib}}} \quad \text { and } \quad S^{\kappa}=\mathrm{e}^{\lambda \kappa \Delta_{\mathrm{fib}}}
$$

and the fibrewise products

$$
a \circ_{\kappa} b:=S^{\kappa}\left(S^{-\kappa} a \circ_{\text {Weyl }} S^{-\kappa} b\right)=\mu \circ \mathrm{e}^{(\kappa+1) \lambda P+(\kappa-1) \lambda \bar{P}} a \otimes b .
$$

Clearly $\circ_{\kappa}$ is again a fibrewise associative deformation of $\mu$ and fibrewisely equivalent to $\circ_{\text {Weyl }}$ via $S^{\kappa}$. Besides the Weyl ordered case $(\kappa=0)$ the Wick ordered case $(\kappa=1)$ and the anti Wick ordered case $(\kappa=-1)$ are of particular interest as here $\bar{P}$ respectively $P$ are absent in (16). We shall denote these fibrewise products by $\circ_{\text {Wick }}$ and $\circ \overline{\text { Wick }}$.

We also observe that the bimodule structure of $\mathcal{W} \otimes \Lambda^{\bullet} \otimes \mathcal{E}$ can be deformed yielding a bimodule structure with respect to the deformed products $\circ_{\kappa}$ of $\mathcal{W} \otimes \Lambda^{\bullet} \otimes \mathcal{E} n d(\mathcal{E})$ and $\mathcal{W} \otimes \Lambda^{\bullet}$. We shall denote the corresponding bimodule multiplications by $\circ_{\kappa}$ as well. 
Remark 1 It is clear that $\Delta_{\mathrm{fib}}, P, \bar{P}$, and $\circ_{\kappa}$ can be defined as soon as one has an almost complex structure compatible with the symplectic structure on an arbitrary symplectic manifold. In principle one can carry through Fedosov's construction in this case as well [25]. However, the resulting star products $\star_{\kappa}$ seem to have no particular properties like 'separation of variables' for the cases $\kappa= \pm 1$. This only will happen on a (semi-) Kähler manifold.

We finally mention some further relations between the various operators. First note that $\left[\Delta_{\text {fib }}, \delta\right]=0$ whence $S^{\kappa} \delta S^{-\kappa}=\delta$ for all $\kappa$. Moreover, since $\nabla$ is the Kähler connection we have $\left[\Delta_{\mathrm{fib}}, D\right]=0$ as well as $\left[\Delta_{\mathrm{fib}}, D^{E}\right]=0$ and $\left[\Delta_{\mathrm{fib}}, D^{\prime}\right]=0$. This implies $S^{\kappa} D S^{-\kappa}=D$ and hence $D$ is a $\circ_{\kappa}$-derivation as well. Analogously, $D^{\prime}$ and $D^{E}$ are (module-) derivations with respect to $\circ_{\kappa}$. The following lemma is a slight variation of [6, Prop. 4.1] and [17, Sect. 5.3]:

Lemma 1 We have $S^{\kappa} R=R+\kappa \lambda \Delta_{\mathrm{fib}} R=R+\kappa \lambda \varrho$ and hence

$$
D^{2}=\frac{\mathrm{i}}{\lambda} \operatorname{ad}_{\kappa}\left(S^{\kappa} R\right)=\frac{\mathrm{i}}{\lambda} \operatorname{ad}_{\kappa}(R),
$$

as the Ricci form $\varrho$, see (89), is central with respect to $\circ_{\kappa}$. Moreover, $S^{\kappa} R^{E}=R^{E}$ and hence

$$
\left(D^{E}\right)^{2}=\frac{\mathrm{i}}{\lambda} \operatorname{ad}_{\kappa}(R)+R^{E} \quad \text { and } \quad\left(D^{\prime}\right)^{2}=\frac{\mathrm{i}}{\lambda} \operatorname{ad}_{\kappa}\left(R-\mathrm{i} \lambda R^{E}\right) .
$$

\section{Fedosov star products and deformed vector bundles}

Using the results of the previous section as well as the standard arguments of Fedosov's construction [16, 35] we easily arrive at the following theorem:

Theorem 1 We fix $\kappa \in \mathbb{R}$. For any series of closed two-forms $\Omega_{\kappa} \in \lambda \Gamma^{\infty}\left(\bigwedge^{2} T^{*} M\right)[[\lambda]]$ there exist unique $r_{\kappa} \in \mathcal{W} \otimes \Lambda^{1}, r_{\kappa}^{\prime} \in \mathcal{W} \otimes \Lambda^{1} \otimes \mathcal{E} n d(\mathcal{E})$ of total degree $\geq 3$ such that

$$
\delta r_{\kappa}=R+D r_{\kappa}+\frac{\mathrm{i}}{\lambda} r_{\kappa} \circ_{\kappa} r_{\kappa}+\Omega_{\kappa} \quad \text { and } \quad \delta^{-1} r_{\kappa}=0
$$

and

$$
\delta r_{\kappa}^{\prime}=R-\mathrm{i} \lambda R^{E}+D^{\prime} r_{\kappa}^{\prime}+\frac{\mathrm{i}}{\lambda} r_{\kappa}^{\prime} \circ_{\kappa} r_{\kappa}^{\prime}+\Omega_{\kappa} \quad \text { and } \quad \delta^{-1} r_{\kappa}^{\prime}=0
$$

In this case the super derivations

$$
\mathcal{D}_{\kappa}=-\delta+D+\frac{\mathrm{i}}{\lambda} \operatorname{ad}_{\kappa}\left(r_{\kappa}\right) \quad \text { and } \quad \mathcal{D}_{\kappa}^{\prime}=-\delta+D^{\prime}+\frac{\mathrm{i}}{\lambda} \operatorname{ad}_{\kappa}\left(r_{\kappa}^{\prime}\right)
$$

have square zero. The maps

$$
\sigma: \operatorname{ker} \mathcal{D}_{\kappa} \cap \mathcal{W} \otimes \Lambda^{0} \rightarrow C^{\infty}(M)[[\lambda]] \quad \text { and } \quad \sigma: \operatorname{ker} \mathcal{D}_{\kappa}^{\prime} \cap \mathcal{W} \otimes \Lambda^{0} \otimes \mathcal{E} n d(\mathcal{E}) \rightarrow \Gamma^{\infty}(\operatorname{End}(E))[[\lambda]]
$$

are $\mathbb{C}[[\lambda]]$-linear bijections with inverses denoted by $\tau_{\kappa}$ and $\tau_{\kappa}^{\prime}$, respectively. Finally,

$$
f \star_{\kappa} g=\sigma\left(\tau_{\kappa}(f) \circ_{\kappa} \tau_{\kappa}(g)\right) \quad \text { and } \quad A \star_{\kappa}{ }^{\prime} B=\sigma\left(\tau_{\kappa}^{\prime}(A) \circ_{\kappa} \tau_{\kappa}^{\prime}(B)\right)
$$

define associative deformations of $C^{\infty}(M)$ and $\Gamma^{\infty}(\operatorname{End}(E))$, respectively, and $\star_{\kappa}$ is a star product with characteristic class

$$
c\left(\star_{\kappa}\right)=\frac{1}{\mathrm{i} \lambda}\left([\omega]+\left[\Omega_{\kappa}\right]-\kappa \lambda[\varrho]\right) .
$$


Proof: The proof is completely standard and follows from [16, 35] with some obvious modifications. We only indicate the computation of the characteristic class: We define $r=S^{-\kappa} r_{\kappa}$ whence clearly

$$
\delta r=R-\kappa \lambda \varrho+D r+\frac{\mathrm{i}}{\lambda} r \circ_{\text {Weyl }} r+\Omega_{\kappa}
$$

using Lemma 1 and $\Delta_{\mathrm{fib}} \Omega_{\kappa}=0$. Moreover, $\delta^{-1} r$ is some element of total degree $\geq 3$. Thus the characteristic class of the Weyl ordered Fedosov star product $\star$ built out of $r$ is simply given by the expression in (24), see e.g. [31. But $\star$ and $\star_{\kappa}$ are equivalent as their Fedosov derivatives are fibrewisely conjugate by $S^{\kappa}$, see [闰, Prop. 1].

For the deformed bimodule structure of $\Gamma^{\infty}(E)$ with respect to $\star_{\kappa}{ }^{\prime}$ and $\star_{\kappa}$ we proceed completely analogously to [35]. First we define

$$
r_{\kappa}^{E}:=\frac{\mathrm{i}}{\lambda}\left(r_{\kappa}^{\prime}-r_{\kappa}\right)
$$

which is a well-defined formal power series in $\lambda$ as $r_{\kappa}^{\prime}$ and $r_{\kappa}$ coincide in zeroth order of $\lambda$. Then we define $\mathcal{D}_{\kappa}^{E}: \mathcal{W} \otimes \Lambda^{\bullet} \otimes \mathcal{E} \rightarrow \mathcal{W} \otimes \Lambda^{\bullet+1} \otimes \mathcal{E}$ by

$$
\mathcal{D}_{\kappa}^{E}=-\delta+D^{E}+\frac{\mathrm{i}}{\lambda} \operatorname{ad}_{\kappa}\left(r_{\kappa}\right)+r_{\kappa}^{E}
$$

where $\operatorname{ad}_{\kappa}\left(r_{\kappa}\right)$ is defined as usual and $r_{\kappa}^{E}$ acts by $\circ_{\kappa}$-left multiplications. The following construction is an immediate adaption of [35, Thm. 3] to our $\kappa$-ordered situation:

Theorem 2 The Fedosov derivative $\mathcal{D}_{\kappa}^{E}$ satisfies

$$
\begin{aligned}
& \mathcal{D}_{\kappa}^{E}\left(\Psi \circ_{\kappa} b\right)=\mathcal{D}_{\kappa}^{E} \Psi \circ_{\kappa} b+(-1)^{k} \Psi \circ_{\kappa} \mathcal{D}_{\kappa} b, \\
& \mathcal{D}_{\kappa}^{E}\left(a \circ_{\kappa} \Psi\right)=\mathcal{D}_{\kappa}^{\prime} a \circ_{\kappa} \Psi+(-1)^{\ell} a \circ_{\kappa} \mathcal{D}_{\kappa}^{E} \Psi,
\end{aligned}
$$

and $\left(\mathcal{D}_{\kappa}^{E}\right)^{2}=0$, for $a \in \mathcal{W} \otimes \Lambda^{\ell} \otimes \mathcal{E} n d(\mathcal{E}), \Psi \in \mathcal{W} \otimes \Lambda^{k} \otimes \mathcal{E}$, and $b \in \mathcal{W} \otimes \Lambda^{\bullet}$. Moreover, $\sigma:$ $\operatorname{ker} \mathcal{D}_{\kappa}^{E} \cap \mathcal{W} \otimes \Lambda^{0} \otimes \mathcal{E} \rightarrow \Gamma^{\infty}(E)[[\lambda]]$ is a $\mathbb{C}[[\lambda]]$-linear bijection with inverse denoted by $\tau_{\kappa}^{E}$. Finally,

$$
\begin{aligned}
& A \bullet_{\kappa}^{\prime} s:=\sigma\left(\tau_{\kappa}^{\prime}(A) \circ_{\kappa} \tau_{\kappa}^{E}(s)\right) \\
& s \bullet_{\kappa} f:=\sigma\left(\tau_{\kappa}^{E}(s) \circ_{\kappa} \tau_{\kappa}(f)\right)
\end{aligned}
$$

defines $a \star_{\kappa}{ }^{\prime}-\star_{\kappa}$ bimodule deformation of $\Gamma^{\infty}(E)$.

Remark 2 i.) In the particular case of a line bundle $E=L$ we obtain a star product $\star^{\prime}$ for $C^{\infty}(M)[[\lambda]]=\Gamma^{\infty}(\operatorname{End}(L))[[\lambda]]$ with a corresponding bimodule deformation. Then the characteristic class of $\star^{\prime}{ }_{\kappa}$ is given by

$$
c\left(\star_{\kappa}^{\prime}\right)=\frac{1}{\mathrm{i} \lambda}\left([\omega]+\left[\Omega_{\kappa}\right]-\kappa \lambda[\varrho]\right)+2 \pi \mathrm{i} c_{1}(L),
$$

where $c_{1}(L)$ is the Chern class of L. This follows either from [9, Thm. 3.1] or by an analogous argument as in [35, Cor. 2].

ii.) In general, $\star_{\kappa}^{\prime}$ and $\star_{\kappa}$ are Morita equivalent deformations and $\left(\Gamma^{\infty}(E)[[\lambda]], \bullet_{\kappa}^{\prime}, \bullet_{\kappa}\right)$ is a Morita equivalence bimodule [39, Prop. 1]. 


\section{The Wick Type Properties of $\star_{\text {Wick }}, \star_{\text {Wick }}^{\prime}, \bullet_{\text {Wick }}$ and $\bullet_{\text {Wick }}^{\prime}$}

In this section we shall consider the deformations $\star_{\kappa}, \star^{\prime}{ }_{\kappa}$ and the bimodule structures $\bullet_{\kappa}, \bullet^{\prime}{ }_{\kappa}$ more closely in the case $\kappa=1$. Actually an analogous consideration can also be carried out in the case $\kappa=-1$ by almost trivial generalizations of the given results. We set $\Omega=\Omega_{1}=\Omega_{\text {Wick }}$.

First we need some additional notations that make use of the complex structure that enable us to consider the splittings into holomorphic and anti-holomorphic part of the mappings involved in the Fedosov construction. For a detailed discussion of this topic the reader is referred to [32, Appx. A]. By $\pi_{z}$ we denote the projection onto the holomorphic form part in the symmetric and the anti-symmetric part of $\mathcal{W} \otimes \Lambda^{\bullet}$. Then $\pi_{z}$ naturally extends to a projection defined on $\mathcal{W} \otimes \Lambda^{\bullet} \otimes \mathcal{E} n d(\mathcal{E})$ and $\mathcal{W} \otimes \Lambda^{\bullet} \otimes \mathcal{E}$ as well. Analogously $\pi_{\bar{z}}$ denotes the projection onto the antiholomorphic form part of $\mathcal{W} \otimes \Lambda^{\bullet}$ that also extends naturally to $\mathcal{W} \otimes \Lambda^{\bullet} \otimes \mathcal{E} n d(\mathcal{E})$ and $\mathcal{W} \otimes \Lambda^{\bullet} \otimes \mathcal{E}$. Obviously we have $\sigma=\pi_{z} \pi_{\bar{z}}=\pi_{\bar{z}} \pi_{z}$. From the very definitions of the products and the fibrewise bimodule multiplications ${ }^{{ }_{\text {Wick }}}$ it is easy to see that

$$
\pi_{z}\left(F \circ_{\text {Wick }} G\right)=\pi_{z}\left(\left(\pi_{z} F\right) \circ_{\text {Wick }} G\right) \quad \text { and } \quad \pi_{\bar{z}}\left(F \circ_{\text {Wick }} G\right)=\pi_{\bar{z}}\left(F \circ_{\text {Wick }}\left(\pi_{\bar{z}} G\right)\right),
$$

where $F, G$ are elements in $\mathcal{W} \otimes \Lambda^{\bullet}, \mathcal{W} \otimes \Lambda^{\bullet} \otimes \mathcal{E}$ or $\mathcal{W} \otimes \Lambda^{\bullet} \otimes \mathcal{E} n d(\mathcal{E})$ such that the muliplications make sense. Using a local holomorphic chart of $M$ it is easy to see that $\delta=\delta_{z}+\delta_{\bar{z}}, \delta^{*}=\delta_{z}^{*}+\delta_{\bar{z}}^{*}$, $D=D_{z}+D_{\bar{z}}, D^{E}=D_{z}^{E}+D_{\bar{z}}^{E}$ and $D^{\prime}=D_{z}^{\prime}+D_{\bar{z}}^{\prime}$, where for instance $D_{z}^{\prime}(f \otimes \alpha \otimes A):=$ $\nabla_{Z_{i}} f \otimes d z^{i} \wedge \alpha \otimes A+f \otimes \partial \alpha \otimes A+f \otimes d z^{i} \wedge \alpha \otimes \nabla_{Z_{i}}^{\operatorname{End}(E)} A$ and $D_{\bar{z}}^{\prime}(f \otimes \alpha \otimes A):=\nabla_{\bar{Z}_{i}} f \otimes$ $d \bar{z}^{i} \wedge \alpha \otimes A+f \otimes \bar{\partial} \alpha \otimes A+f \otimes d \bar{z}^{i} \wedge \alpha \otimes \nabla_{\bar{Z}_{i}}^{\operatorname{End}(E)} A$. The other splittings are defined similarly. Completely analogously to the definition of $\delta^{-1}$ one defines $\delta_{z}^{-1}$ for $a$ with $d z^{i} \vee i_{s}\left(Z_{i}\right) a=k a$ and $d z^{i} \wedge i_{a}\left(Z_{i}\right) a=l a$ by $\delta_{z}^{-1} a:=\frac{1}{k+l} \delta_{z}^{*} a$ in case $k+l \neq 0$ and $\delta_{z}^{-1} a:=0$ in case $k+l=0 . \delta_{\bar{z}}^{-1}$ is defined in the analogous way and an easy computation yields the following decompositions:

$$
\delta_{z}^{-1} \delta_{z}+\delta_{z} \delta_{z}^{-1}+\pi_{\bar{z}}=\mathrm{id} \text { and } \delta_{\bar{z}}^{-1} \delta_{\bar{z}}+\delta_{\bar{z}} \delta_{\bar{z}}^{-1}+\pi_{z}=\mathrm{id} .
$$

Furthermore we have the relations: $\pi_{z} \delta=\delta_{z} \pi_{z}, \pi_{z} \delta^{-1}=\delta_{z}^{-1} \pi_{z}, \pi_{z} D=D_{z} \pi_{z}, \pi_{z} D^{E}=D_{z}^{E} \pi_{z}$ and $\pi_{z} D^{\prime}=D_{z}^{\prime} \pi_{z}$ and analogous formulas with $\bar{z}$ instead of $z$. Using the equation $\delta^{2}=0$, the fact that $\delta$ super-commutes with $D, D^{E}$ and $D^{\prime}$ and the equations for $D^{2}, D^{E^{2}}$ and $D^{\prime 2}$ it is easy to derive super-commutation relations for the holomorphic and the anti-holomorphic parts of the involved mappings, see also [32, Lem. 3].

Lemma 2 Let $r_{\text {Wick }}, r_{\text {Wick }}^{\prime}$ and $r_{\text {Wick }}^{E}$ denote the elements of $\mathcal{W} \otimes \Lambda^{1}, \mathcal{W} \otimes \Lambda^{1} \otimes \mathcal{E} n d(\mathcal{E})$ constructed in the preceding section in case $\kappa=1$. Then we have the following:

i.) In case $\Omega$ is of type $(1,1)$ then in addition

$$
\pi_{z} r_{\mathrm{Wick}}=0 \quad \text { and } \quad \pi_{\bar{z}} r_{\mathrm{Wick}}=0 .
$$

ii.) In case $\Omega$ and $R^{E}$ are of type $(1,1)$ then

$$
\pi_{z} r_{\mathrm{Wick}}^{\prime}=\pi_{\bar{z}} r_{\mathrm{Wick}}^{\prime}=0 \quad \text { and } \quad \pi_{z} r_{\mathrm{Wick}}^{E}=\pi_{\bar{z}} r_{\mathrm{Wick}}^{E}=0 .
$$

Proof: The proof of i.) can be found in [32, Lem. 4.1]. Applying $\pi_{z}$ to (20) we obtain

$$
\delta_{z} \pi_{z} r_{\text {Wick }}^{\prime}=D_{z}^{\prime} \pi_{z} r_{\text {Wick }}^{\prime}+\frac{\mathrm{i}}{\lambda} \pi_{z}\left(\left(\pi_{z} r_{\text {Wick }}^{\prime}\right) \circ_{\text {Wick }} r_{\text {Wick }}^{\prime}\right) \quad \text { and } \quad \delta_{z}^{-1} \pi_{z} r_{\text {Wick }}^{\prime}=0
$$

using $\pi_{z} R^{E}=\pi_{z} \Omega=0$. Using the decomposition $\delta_{z}^{-1} \delta_{z}+\delta_{z} \delta_{z}^{-1}+\pi_{\bar{z}}=\mathrm{id}$ this implies that $\pi_{z} r_{\text {Wick }}^{\prime}$ is a fixed point of the mapping $L: \pi_{z}\left(\mathcal{W} \otimes \Lambda^{1} \otimes \mathcal{E} n d(\mathcal{E})\right) \ni a \mapsto \delta_{z}^{-1}\left(D_{z}^{\prime} a+\frac{\mathrm{i}}{\nu} \pi_{z}\left(a \circ_{\text {Wick }} r_{\text {Wick }}^{\prime}\right)\right) \in \pi_{z}\left(\mathcal{W} \otimes \Lambda^{1} \otimes \mathcal{E} n d(\varepsilon)\right)$ 
that raises the total degree at least by one and hence has a unique fixed point. Obviously $L$ has 0 as trivial fixed point implying $\pi_{z} r_{\text {Wick }}^{\prime}=0$ by uniqueness. Analogously one proves that $\pi_{\bar{z}} r_{\text {Wick }}^{\prime}=0$. Then the statement for $r_{\text {Wick }}^{E}$ follows.

From the explicit shape of the fibrewise products and the bimodule multiplications $\circ_{\text {Wick }}$ it is obvious that the products $\star_{\text {Wick }}, \star_{\text {Wick }}^{\prime}$ and the bimodule muliplications $\bullet_{\text {Wick }}, \bullet_{\text {Wick }}^{\prime}$ are completely determined by the knowledge of the projections on the totally holomorphic resp. anti-holomorphic part of the respective Fedosov-Taylor series. Therefore it is worth deriving simpler formulas for these projections in order to investigate the products and the bimodule multiplications.

Proposition 1 Let $R^{E}$ and $\Omega$ be of type $(1,1)$ then the projections of $\tau_{\text {Wick }}(f), \tau_{\text {Wick }}^{\prime}(A)$ and $\tau_{\text {Wick }}^{E}(s)$ for $f \in C^{\infty}(M)[[\lambda]], A \in \Gamma^{\infty}(\operatorname{End}(E))[[\lambda]]$ and $s \in \Gamma^{\infty}(E)[[\lambda]]$ satisfy the equations:

$$
\begin{array}{cccc}
\delta_{z} \pi_{z} \tau_{\text {Wick }}(f)=D_{z} \pi_{z} \tau_{\text {Wick }}(f)-\frac{\mathrm{i}}{\lambda} \pi_{z}\left(\left(\pi_{z} \tau_{\text {Wick }}(f)\right) \circ_{\text {Wick }} r_{\text {Wick }}\right) & \text { and } & \sigma\left(\pi_{z} \tau_{\text {Wick }}(f)\right)=f \\
\delta_{\bar{z}} \pi_{\bar{z}} \tau_{\text {Wick }}(f)=D_{\bar{z}} \pi_{\bar{z}} \tau_{\text {Wick }}(f)+\frac{\mathrm{i}}{\lambda} \pi_{\bar{z}}\left(r_{\text {Wick }} \circ_{\text {Wick }}\left(\pi_{\bar{z}} \tau_{\text {Wick }}(f)\right)\right) & \text { and } & \sigma\left(\pi_{\bar{z}} \tau_{\text {Wick }}(f)\right)=f \\
\delta_{z} \pi_{z} \tau_{\text {Wick }}^{\prime}(A)=D_{z}^{\prime} \pi_{z} \tau_{\text {Wick }}^{\prime}(A)-\frac{\mathrm{i}}{\lambda} \pi_{z}\left(\left(\pi_{z} \tau_{\text {Wick }}^{\prime}(A)\right) \circ_{\text {Wick }} r_{\text {Wick }}^{\prime}\right) & \text { and } & \sigma\left(\pi_{z} \tau_{\text {Wick }}^{\prime}(A)\right)=A \\
\delta_{\bar{z}} \pi_{\bar{z}} \tau_{\text {Wick }}^{\prime}(A)=D_{\bar{z}}^{\prime} \pi_{\bar{z}} \tau_{\text {Wick }}^{\prime}(A)+\frac{\mathrm{i}}{\lambda} \pi_{\bar{z}}\left(r_{\text {Wick }}^{\prime} \circ_{\text {Wick }}\left(\pi_{\bar{z}} \tau_{\text {Wick }}^{\prime}(A)\right)\right) & \text { and } & \sigma\left(\pi_{\bar{z}} \tau_{\text {Wick }}^{\prime}(A)\right)=A \\
\delta_{z} \pi_{z} \tau_{\text {Wick }}^{E}(s)=D_{z}^{E} \pi_{z} \tau_{\text {Wick }}^{E}(s)-\frac{\mathrm{i}}{\lambda} \pi_{z}\left(\left(\pi_{z} \tau_{\text {Wick }}^{E}(s)\right) \circ_{\text {Wick }} r_{\text {Wick }}\right) & \text { and } & \sigma\left(\pi_{z} \tau_{\text {Wick }}^{E}(s)\right)=s \\
\delta_{\bar{z}} \pi_{\bar{z}} \tau_{\text {Wick }}^{E}(s)=D_{\bar{z}}^{E} \pi_{\bar{z}} \tau_{\text {Wick }}^{E}(s)+\frac{\mathrm{i}}{\lambda} \pi_{\bar{z}}\left(r_{\text {Wick }}^{\prime} \circ_{\text {Wick }}\left(\pi_{\bar{z}} \tau_{\text {Wick }}^{E}(s)\right)\right) & \text { and } & \sigma\left(\pi_{\bar{z}} \tau_{\text {Wick }}^{E}(s)\right)=s
\end{array}
$$

from which they are uniquely determined.

Proof: The proof of equations (37) and (38) again can be found in [32, Prop. 4.3]. It is easy to see that this proof can be slightly modified to obtain the remaining statements of the proposition.

As an easy consequence of the preceding proposition we find:

Lemma 3 Let $\mathcal{U} \subseteq M$ be an open subset of $M$ and let $R^{E}$ and $\Omega$ be of type $(1,1)$.

i.) For all $f \in C^{\infty}(M)$ anti-holomorphic on $\mathcal{U}$ we have $\left.\pi_{z} \tau_{\text {Wick }}(f)\right|_{\mathfrak{U}}=\left.f\right|_{\mathfrak{u}}$.

ii.) For all $g \in C^{\infty}(M)$ holomorphic on $\mathcal{U}$ we have $\left.\pi_{\bar{z}} \tau_{\text {Wick }}(g)\right|_{\mathfrak{u}}=\left.g\right|_{\mathfrak{u}}$.

iii.) For all $A \in \Gamma^{\infty}(\operatorname{End}(E))$ such that $\left.\nabla_{Y}^{\operatorname{End}(E)} A\right|_{\mathcal{u}}=0$ for all $Y \in \Gamma^{\infty}\left(T M^{1,0}\right)$ we have $\left.\pi_{z} \tau_{\text {Wick }}^{\prime}(A)\right|_{\text {u }}=\left.A\right|_{U}$.

iv.) For all $B \in \Gamma^{\infty}(\operatorname{End}(E))$ such that $\left.\nabla_{X}^{\operatorname{End}(E)} B\right|_{\mathfrak{u}}=0$ for all $X \in \Gamma^{\infty}\left(T M^{0,1}\right)$ we have $\left.\pi_{\bar{z}} \tau_{\text {Wick }}^{\prime}(B)\right|_{u}=\left.B\right|_{u}$.

v.) For all $s \in \Gamma^{\infty}(E)$ such that $\left.\nabla_{Y}^{E} s\right|_{\mathfrak{u}}=0$ for all $Y \in \Gamma^{\infty}\left(T M^{1,0}\right)$ we have $\left.\pi_{z} \tau_{\text {wick }}^{E}(s)\right|_{\mathfrak{u}}=\left.s\right|_{\mathfrak{u}}$.

vi.) For all $t \in \Gamma^{\infty}(E)$ such that $\left.\nabla_{X}^{E} t\right|_{\mathfrak{u}}=0$ for all $X \in \Gamma^{\infty}\left(T M^{0,1}\right)$ we have $\left.\pi_{\bar{z}} \tau_{\text {Wick }}^{E}(t)\right|_{\mathfrak{u}}=\left.t\right|_{u}$.

PROOF: To prove these assertions one just has to show that the given expressions for the projections of the respective Fedosov-Taylor series solve the equations given in Proposition 1 which is an easy task using 
that the projections of $r_{\text {Wick }}$ and $r_{\text {Wick }}^{\prime}$ onto the totally holomorphic and anti-holomorphic form part vanish.

From the results of the preceding lemma we can deduce that the products $\star_{\text {Wick }}, \star_{\text {Wick }}^{\prime}$ and the bimodule multiplications $\bullet_{\text {Wick }}, \bullet_{\text {Wick }}^{\prime}$ have the following Wick type properties:

Theorem 3 Let $\mathcal{U} \subseteq M$ be an open subset of $M$ and let $R^{E}$ and $\Omega$ be of type $(1,1)$.

i.) For all $s \in \Gamma^{\infty}(E), f, g \in C^{\infty}(M)$ such that $g$ is holomorphic in $\mathcal{U}$ we have

$$
\left.s \bullet_{\text {Wick }} g\right|_{u}=\left.s g\right|_{u} \quad \text { and }\left.\quad f \star_{\text {Wick }} g\right|_{u}=\left.f g\right|_{\mathfrak{u}} \text {. }
$$

ii.) For all $s \in \Gamma^{\infty}(E), A, B \in \Gamma^{\infty}(\operatorname{End}(E))$ such that $\left.\nabla_{Y}^{\operatorname{End}(E)} B\right|_{\mathcal{u}}=0$ for all $Y \in \Gamma^{\infty}\left(T M^{1,0}\right)$ we have

$$
\left.B \bullet_{\text {Wick }}^{\prime} s\right|_{\mathfrak{U}}=\left.B s\right|_{\mathfrak{U}} \quad \text { and }\left.\quad B \star_{\text {Wick }}^{\prime} A\right|_{\mathfrak{U}}=\left.B A\right|_{\mathfrak{u}} \cdot
$$

iii.) For all $t, s \in \Gamma^{\infty}(E), A \in \Gamma^{\infty}(\operatorname{End}(E)), f \in C^{\infty}(M)$ such that $\left.\nabla_{X}^{E} t\right|_{u}=0$ for all $X \in$ $\Gamma^{\infty}\left(T M^{0,1}\right)$ and $\left.\nabla_{Y}^{E} s\right|_{\mathrm{u}}=0$ for all $Y \in \Gamma^{\infty}\left(T M^{1,0}\right)$ we have

$$
\left.A \bullet_{\text {Wick }}^{\prime} t\right|_{u}=\left.A t\right|_{u} \quad \text { and }\left.\quad s \bullet_{\text {Wick }} f\right|_{u}=\left.s f\right|_{u} \cdot
$$

iv. ) For all $A, B \in \Gamma^{\infty}(\operatorname{End}(E)), f, g \in C^{\infty}(M)$ such that $\left.\nabla_{X}^{\operatorname{End}(E)} B\right|_{\mathcal{u}}=0$ for all $X \in \Gamma^{\infty}\left(T M^{0,1}\right)$ and $g$ anti-holomorphic on $\mathcal{U}$ we have

$$
\left.A \star_{\text {Wick }}^{\prime} B\right|_{\mathfrak{u}}=\left.A B\right|_{\mathfrak{u}} \quad \text { and }\left.\quad g \star_{\text {Wick }} f\right|_{\mathfrak{u}}=\left.g f\right|_{u} \cdot
$$

To conclude this section we want to discuss some more concrete situations where the precondition that the curvature endomorphism $R^{E}$ is of type $(1,1)$ naturally occurs. If $(E, h)$ is a (anti-) holomorphic vector bundle with Hermitian fibre metric then there exists a unique connection $\nabla^{E}$ which is compatible with the (anti-) holomorphic structure as well as with the Hermitian fibre metric (cf. Appendix A). In this case it is known that $R^{E}$ is of type $(1,1)$. Moreover, in this case the conditions of the preceding Proposition under which the products and the bimodule multiplications are just the pointwise ones just mean that the respective sections resp. functions are locally holomorphic resp. anti-holomorphic. In the case of a holomorphic vector bundle $(E, h)$ a section $s$ is called locally anti-holomorphic on $\mathcal{U}$ if the section $s^{b}$ of the dual bundle $E^{*}$ which is also a holomorphic vector bundle defined by $s^{b}\left(s^{\prime}\right):=h\left(s, s^{\prime}\right)$ is locally holomorphic on U. Similarly a section $A \in \Gamma^{\infty}(\operatorname{End}(E))$ is called locally anti-holomorphic if the section $A^{*}$ defined by $h\left(A^{*} s, s^{\prime}\right)=$ $h\left(s, A s^{\prime}\right)$ for all $s, s^{\prime} \in \Gamma^{\infty}(E)$ is locally holomorphic. In the case of an anti-holomorphic vector bundle $(E, h)$ one proceeds similarly to define the notion of locally holomorphic sections.

\section{Local Expressions for the Bimodule Multiplications $\bullet_{\text {Wick }}, \bullet_{\text {Wick }}^{\prime}$}

In this section we want to consider the bimodule multiplications constructed above in the case of a (anti-) holomorphic vector bundle $E$ of fibre dimension $k$ equipped with a connection that is compatible with the (anti-) holomorphic structure and the curvature of which is of type $(1,1)$. We denote by $\left\{\mathcal{U}_{\alpha}\right\}$ a good open cover of $M$. 
Proposition 2 In case $E$ is an anti-holomorphic vector bundle and $e_{\alpha}$ is an anti-holomorphic frame on $\mathfrak{U}_{\alpha}$ one has

$$
\left.\pi_{z} \tau_{\text {Wick }}^{E}(s)\right|_{\mathcal{U}_{\alpha}}=e_{\alpha} \pi_{z} \tau_{\text {Wick }}\left(s_{\alpha}\right)=\pi_{z} \tau_{\text {Wick }}\left(s_{\alpha}^{i}\right) \otimes e_{\alpha, i}
$$

where $s \in \Gamma^{\infty}(E)[[\lambda]]$ has been written as $\left.s\right|_{\mathcal{U}_{\alpha}}=e_{\alpha} s_{\alpha}=e_{\alpha, i} s_{\alpha}^{i}$. Hence we have for all $f \in$ $C^{\infty}(M)[[\lambda]]$

$$
\left.s \bullet_{\text {Wick }} f\right|_{\mathcal{U}_{\alpha}}=e_{\alpha}\left(s_{\alpha} \star_{\text {Wick }} f\right)=e_{\alpha, i}\left(s_{\alpha}^{i} \star_{\text {Wick }} f\right) .
$$

Conversely, $\bullet_{\text {Wick }}$ is globally well-defined by equation (48) and thus it is completely determined by $\star_{\text {Wick }}$.

Proof: To prove that $\pi_{z} \tau_{\text {Wick }}^{E}(s)$ is locally given by $\pi_{z} \tau_{\text {Wick }}\left(s_{\alpha}^{i}\right) \otimes e_{\alpha, i}$ it is enough to show that this expression solves the equations (41) what is easily done using that the frame $e_{\alpha}$ is anti-holomorphic. But then (48) is immediate from the definition of $\bullet_{\text {wick }}$. It remains to show that $\bullet_{\text {wick }}$ is globally defined by this equation. To this end let $e_{\beta}=e_{\alpha} \phi_{\alpha \beta}$ another anti-holomorphic frame where $\phi_{\alpha \beta}$ denotes the anti-holomorphic transition matrix on $\mathcal{U}_{\alpha} \cap \mathcal{U}_{\beta}$ as in Appendix A. We find

$$
e_{\alpha}\left(s_{\alpha} \star_{\text {Wick }} f\right)=e_{\beta} \phi_{\beta \alpha}\left(\left(\phi_{\alpha \beta} s_{\beta}\right) \star_{\text {Wick }} f\right)=e_{\beta}\left(s_{\beta} \star_{\text {Wick }} f\right)
$$

on $\mathcal{U}_{\alpha} \cap \mathcal{U}_{\beta}$, where we have used that the star product $\star_{\text {Wick }}$ is of Wick type implying $\left(\phi_{\alpha \beta} s_{\beta}\right) \star_{\text {Wick }} f=$ $\phi_{\alpha \beta}\left(s_{\beta} \star_{\text {Wick }} f\right)$ since the entries of $\phi_{\alpha \beta}$ are anti-holomorphic.

Remark 3 In fact the preceding proposition states that for an anti-holomorphic vector bundle $E$ with a connection that is compatible with the anti-holomorphic structure the right module multiplication $\bullet_{\text {Wick }}$ is canonical in the sense that is independent of the connection. Actually the statement of the proposition could also be proved without using the local formula for $\pi_{z} \tau_{\text {Wick }}^{E}(s)$ but only the Wick type properties of $\bullet_{\text {Wick }}$ and $\star_{\text {Wick }}$ according to Theorem S. To do so observe that $\Gamma^{\infty}(E)[[\lambda]] \ni s=e_{\alpha} s_{\alpha}=e_{\alpha} \bullet$ Wick $s_{\alpha}$ since $e_{\alpha}$ is an anti-holomorphic frame. But then

$$
\left.s \bullet_{\text {Wick }} f\right|_{\mathfrak{U}_{\alpha}}=\left(e_{\alpha} \bullet_{\text {Wick }} s_{\alpha}\right) \bullet_{\text {Wick }} f=e_{\alpha} \bullet_{\text {Wick }}\left(s_{\alpha} \star_{\text {Wick }} f\right)=e_{\alpha}\left(s_{\alpha} \star_{\text {Wick }} f\right) .
$$

To see that this yields a global definition for $\bullet_{\text {Wick }}$ we note the following equations that are valid on $\mathcal{U}_{\alpha} \cap \mathcal{U}_{\beta}$

$$
\begin{gathered}
e_{\beta}=e_{\alpha} \phi_{\alpha \beta}=e_{\alpha} \bullet_{\text {Wick }} \phi_{\alpha \beta} \quad \text { and } \quad s_{\alpha}=\phi_{\alpha \beta} s_{\beta}=\phi_{\alpha \beta} \star_{\text {Wick }} s_{\beta} \\
\phi_{\alpha \beta} \phi_{\beta \alpha}=\phi_{\alpha \beta} \star_{\text {Wick }} \phi_{\beta \alpha}=\text { id } \quad \text { and } \quad \phi_{\alpha \beta} \phi_{\beta \gamma} \phi_{\gamma \alpha}=\phi_{\alpha \beta} \star_{\text {Wick }} \phi_{\beta \gamma} \star_{\text {Wick }} \phi_{\gamma \alpha}=\text { id, }
\end{gathered}
$$

where $\phi_{\alpha \beta}$ denotes the classical transition matrices, which imply the statement using [36, Lem. 9.3].

As an immediate consequence of these observations we have:

Corollary 1 Let $E$ be an anti-holomorphic vector bundle and let $\tilde{\bullet}_{\text {Wick }}$ be a $\star_{\text {Wick }}$ right module multiplication on $\Gamma^{\infty}(E)[[\lambda]]$ that has the Wick type property, then $\tilde{\bullet}_{\text {Wick }}$ coincides with $\bullet_{\text {Wick }}$.

In contrast the bimodule multiplication $\bullet_{\text {Wick }}^{\prime}$ drastically simplifies in case $E$ is a holomorphic vector bundle, but even in this case it actually depends on the connection $\nabla^{E}$ and hence is not canonical like $\bullet_{\text {Wick }}$. Nevertheless it is completely determined by the product $\star_{\text {Wick }}^{\prime}$ as we state it in the following proposition. 
Proposition 3 In case $E$ is a holomorphic vector bundle and $e_{\alpha}$ is a holomorphic frame and $e^{\alpha}$ is the dual frame of the vector bundle $E^{*}$ on $\mathcal{U}_{\alpha}$ one has

$$
\left.\pi_{\bar{z}} \tau_{\text {Wick }}^{E}(s)\right|_{\mathcal{U}_{\alpha}}=\frac{1}{k} \pi_{\bar{z}} \tau_{\text {Wick }}^{\prime}\left(s \otimes e^{\alpha, i}\right) e_{\alpha, i}
$$

for all $s \in \Gamma^{\infty}(E)[[\lambda]]$. Hence we have for all $A \in \Gamma^{\infty}(\operatorname{End}(E))[[\lambda]]$

$$
\left.A \bullet_{\text {Wick }}^{\prime} s\right|_{\mathrm{U}_{\alpha}}=\frac{1}{k}\left(A \star_{\text {Wick }}^{\prime}\left(s \otimes e^{\alpha, i}\right)\right) e_{\alpha, i}
$$

Moreover, $\bullet_{\text {Wick }}^{\prime}$ is globally well-defined by equation (58) and thus it is completely determined by $\star_{\text {Wick }}^{\prime}$.

Proof: An easy computation using that $e^{\alpha}$ is a holomorphic frame of $E^{*}$ shows that the expression given in (51) solves the equations (42). But then the local formula (52) follows immediately. In fact this could also be proved directly just using the Wick type property of $\star_{\text {Wick }}^{\prime}$ and $\bullet_{\text {Wick }}^{\prime}$. To see this we observe that on $\mathcal{U}_{\alpha}$ we have

$$
s=\frac{1}{k}\left(s \otimes e^{\alpha, i}\right) e_{\alpha, i}=\frac{1}{k}\left(s \otimes e^{\alpha, i}\right) \bullet_{\text {Wick }}^{\prime} e_{\alpha, i}
$$

since $e_{\alpha}$ is a holomorphic frame. But this implies that on $\mathcal{U}_{\alpha}$

$$
\begin{aligned}
A \bullet_{\text {Wick }}^{\prime} s & =\frac{1}{k} A \bullet_{\text {Wick }}^{\prime}\left(\left(s \otimes e^{\alpha, i}\right) \bullet_{\text {Wick }}^{\prime} e_{\alpha, i}\right)=\frac{1}{k}\left(A \star_{\text {Wick }}^{\prime}\left(s \otimes e^{\alpha, i}\right)\right) \bullet_{\text {Wick }}^{\prime} e_{\alpha, i} \\
& =\frac{1}{k}\left(A \star_{\text {Wick }}^{\prime}\left(s \otimes e^{\alpha, i}\right)\right) e_{\alpha, i} .
\end{aligned}
$$

We now write $e_{\alpha, i}=\phi_{\alpha \beta_{i}} e_{\beta, j}$ and $e^{\alpha, i}=\phi^{\alpha \beta_{k}^{i}} e^{\beta, k}$ with $\phi^{\alpha \beta_{r}^{n}} \phi_{\alpha \beta_{i}}^{r}=\delta_{i}^{n}$. Using the Wick type property of $\star_{\text {Wick }}^{\prime}$ and $\bullet_{\text {Wick }}^{\prime}$ and that the transition matrices $\phi_{\alpha \beta}, \phi^{\alpha \beta}$ have holomorphic entries we moreover get

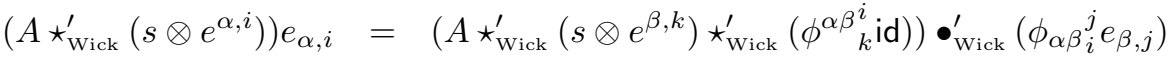

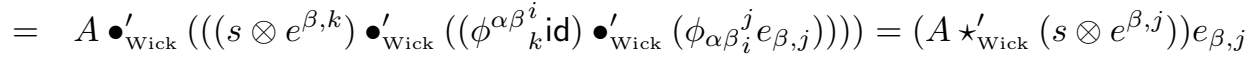

on $\mathcal{U}_{\alpha} \cap \mathcal{U}_{\beta}$ and hence $\bullet_{\text {wick }}^{\prime}$ is globally well-defined by (52).

Corollary 2 Let $E$ be a holomorphic vector bundle and let $\tilde{\bullet}_{\text {Wick }}^{\prime}$ be a $\star_{\text {Wick }}^{\prime}$ left module multiplication on $\Gamma^{\infty}(E)[[\lambda]]$ that has the Wick type property, then $\tilde{\bullet}_{\text {Wick }}^{\prime}$ coincides with $\bullet_{\text {Wick }}^{\prime}$.

\section{Deformed Hermitian Metrics}

In this section we consider a Hermitian fibre metric $h$ for $E$ which is assumed to be either a holomorphic or anti-holomorphic vector bundle. Moreover, let $\nabla^{E}$ be the canonical connection for $(E, h)$. As in the preceding sections we assume $\Omega$ is of type $(1,1)$ and in addition we consider the case where $\Omega$ is real i.e. $\bar{\Omega}=\Omega$. Now $h$ equips $\Gamma^{\infty}(\operatorname{End}(E))$ with a natural ${ }^{*}$-involution defined by $h\left(A s, s^{\prime}\right)=h\left(s, A^{*} s^{\prime}\right)$ for $A \in \Gamma^{\infty}(\operatorname{End}(E))$ and $s, s^{\prime} \in \Gamma^{\infty}(E)$. We extend this involution, together with the complex conjugation, to a super-*-involution of $\mathcal{W} \otimes \Lambda^{\bullet} \otimes \mathcal{E} n d(\mathcal{E})$ and $\mathcal{W} \otimes \Lambda^{\bullet}$, respectively. It is well-known that the fibrewise Wick product $\circ_{\text {Wick }}$ is compatible with this ${ }^{*}$ involution, i.e. $\left(a \circ_{\text {Wick }} b\right)^{*}=(-1)^{\operatorname{deg}_{\mathrm{a}} a \operatorname{deg}_{\mathrm{a}} b} b^{*} \circ_{\text {Wick }} a^{*}$ for all $a, b \in \mathcal{W} \otimes \Lambda^{\bullet} \otimes \mathcal{E} n d(\mathcal{E})$. From the unique characterizations of $r_{\text {Wick }}^{\prime}$ and $r_{\text {Wick }}$ the following lemma is straightforward, see also [35, Lem. 7]. 
Lemma 4 Let $\Omega=\bar{\Omega}$ be real, then

$$
\left(r_{\text {Wick }}^{\prime}\right)^{*}=r_{\text {Wick }}^{\prime}, \quad \overline{r_{\text {Wick }}}=r_{\text {Wick }}, \quad\left(r_{\text {Wick }}^{E}\right)^{*}=-r_{\text {Wick }}^{E}
$$

and hence $\left(\mathcal{D}^{\prime}{ }_{\text {Wick }} a\right)^{*}=\mathcal{D}_{\text {Wick }}^{\prime} a^{*}$ and $\overline{\mathcal{D}_{\text {Wick }} b}=\mathcal{D}_{\text {Wick }} \bar{b}$ for $a \in \mathcal{W} \otimes \Lambda^{\bullet} \otimes \mathcal{E} n d(\mathcal{E})$ and $b \in \mathcal{W} \otimes \Lambda^{\bullet}$. Moreover we have $\left(\tau_{\text {Wick }}^{\prime}(A)\right)^{*}=\tau_{\text {Wick }}^{\prime}\left(A^{*}\right)$ and $\overline{\tau_{\text {Wick }}(f)}=\tau_{\text {Wick }}(\bar{f})$ and hence $\star_{\text {Wick }}^{\prime}$ and $\star_{\text {Wick }}$ are Hermitian deformations, i.e.

$$
\left(A \star_{\text {Wick }}^{\prime} B\right)^{*}=B^{*} \star_{\text {Wick }}^{\prime} A^{*} \text { and } \overline{f \star_{\text {Wick }} g}=\bar{g} \star_{\text {Wick }} \bar{f} \text {. }
$$

In a next step we extend the fibre metric $h$ to a metric on $\mathcal{W} \otimes \Lambda^{\bullet} \otimes \mathcal{E}$ with values in $\mathcal{W} \otimes \Lambda^{\bullet}$ by defining

$$
H\left(f \otimes \alpha \otimes s, g \otimes \beta \otimes s^{\prime}\right):=\bar{f} \circ_{\text {Wick }} g \otimes \bar{\alpha} \wedge \beta h\left(s, s^{\prime}\right)
$$

The following properties are immediate円:

Lemma 5 For $a \in \mathcal{W} \otimes \Lambda^{\bullet} \otimes \mathcal{E} n d(\mathcal{E}), \Psi, \Psi^{\prime} \in \mathcal{W} \otimes \Lambda^{\bullet} \otimes \mathcal{E}$ and $b \in \mathcal{W} \otimes \Lambda^{\bullet}$ we have

$$
\begin{gathered}
H\left(a \circ_{\text {Wick }} \Psi, \Psi^{\prime}\right)=(-1)^{\operatorname{deg}_{\mathrm{a}} a \operatorname{deg}_{\mathrm{a}} \Psi} H\left(\Psi, a^{*} \circ_{\text {Wick }} \Psi^{\prime}\right), \\
H\left(\Psi, \Psi^{\prime} \circ_{\text {Wick }} b\right)=H\left(\Psi, \Psi^{\prime}\right) \circ_{\text {Wick }} b, \\
H\left(\Psi, \Psi^{\prime}\right)=(-1)^{\operatorname{deg}_{\mathrm{a}} \Psi \operatorname{deg}_{\mathrm{a}} \Psi^{\prime}} \overline{H\left(\Psi^{\prime}, \Psi\right)} .
\end{gathered}
$$

Now a simple computation using the preceding two lemmas yields the following compatibility of $\mathcal{D}_{\text {Wick }}$ with $H$

$$
\mathcal{D}_{\text {Wick }}\left(H\left(\Psi, \Psi^{\prime}\right)\right)=H\left(\mathcal{D}_{\text {Wick }}^{E} \Psi, \Psi^{\prime}\right)+(-1)^{\operatorname{deg}_{\mathrm{a}} \Psi} H\left(\Psi, \mathcal{D}_{\text {Wick }}^{E} \Psi^{\prime}\right) .
$$

As a consequence we can define a deformed Hermitian metric $\boldsymbol{h}$ by

$$
\boldsymbol{h}\left(s, s^{\prime}\right):=\sigma\left(H\left(\tau_{\text {Wick }}^{E}(s), \tau_{\text {Wick }}^{E}\left(s^{\prime}\right)\right)\right) \text {. }
$$

Proposition 4 The map $\boldsymbol{h}$ is $\mathbb{C}[[\lambda]]$-linear in the second argument and satisfies

$$
\begin{array}{rll}
\boldsymbol{h}\left(s, s^{\prime}\right)=\overline{\boldsymbol{h}\left(s^{\prime}, s\right)} & \text { and } & \boldsymbol{h}(s, s) \geq 0 \\
\boldsymbol{h}\left(s, s^{\prime} \bullet_{\text {Wick }} f\right) & =\quad \boldsymbol{h}\left(s, s^{\prime}\right) \star_{\text {Wick }} f \\
\boldsymbol{h}\left(A \bullet_{\text {Wick }}^{\prime} s, s^{\prime}\right) & =\quad \boldsymbol{h}\left(s, A^{*} \bullet_{\text {Wick }}^{\prime} s^{\prime}\right)
\end{array}
$$

for all $s, s^{\prime} \in \Gamma^{\infty}(E)[[\lambda]], f \in C^{\infty}(M)[[\lambda]]$ and $A \in \Gamma^{\infty}(\operatorname{End}(E))[[\lambda]]$.

Here the positivity of $\boldsymbol{h}$ is understood in the sense of [8].

From the properties of ${ }_{o_{\text {Wick }}}$ it is obvious that $\boldsymbol{h}\left(s, s^{\prime}\right)=\sigma\left(H\left(\pi_{\bar{z}} \tau_{\text {Wick }}^{E}(s), \pi_{\bar{z}} \tau_{\text {Wick }}^{E}\left(s^{\prime}\right)\right)\right)$ and this implies the following lemma using the statements of Lemma 3 .

\footnotetext{
${ }^{1}$ In 35 an obvious sign was missing.
} 
Lemma 6 Let $\mathcal{U} \subseteq M$ be an open subset of $M$. In case $s$ or $s^{\prime}$ are locally holomorphic sections on u we have

$$
\left.\boldsymbol{h}\left(s, s^{\prime}\right)\right|_{u}=\left.h\left(s, s^{\prime}\right)\right|_{u}
$$

To conclude this section we now want to give an explicit local expression for $\boldsymbol{h}$.

Proposition 5 In case $E$ is an anti-holomorphic vector bundle and $e_{\alpha}$ is an anti-holomorphic frame on $\mathfrak{U}_{\alpha}$ one has

$$
\left.\boldsymbol{h}\left(s, s^{\prime}\right)\right|_{\mathcal{u}_{\alpha}}=\overline{s_{\alpha}^{i}} \star_{\text {Wick }} \boldsymbol{h}\left(e_{\alpha, i}, e_{\alpha, j}\right) \star_{\text {Wick }} s_{\alpha}^{\prime j},
$$

where we have written $\left.s\right|_{\mathfrak{U}_{\alpha}}=e_{\alpha, j} s_{\alpha}^{j}$ and $\left.s^{\prime}\right|_{\mathfrak{U}_{\alpha}}=e_{\alpha, j} s_{\alpha}^{\prime j}$.

Proof: The proof is straightforward using Proposition 4, Lemma 6 and the Wick type property of $\bullet_{\text {Wick }}$.

Remark 4 In fact it is easy to see that the expression for $\boldsymbol{h}\left(s, s^{\prime}\right) \mid u_{\alpha}$ given in the preceding proposition globally defines the deformed metric $\boldsymbol{h}$ by similar arguments as in Section 5 .

Proposition 6 In case $E$ is a holomorphic vector bundle and $e_{\alpha}$ is a holomorphic frame on $\mathcal{U}_{\alpha}$ and $e^{\alpha}$ is the dual frame of the vector bundle $E^{*}$ on $\mathcal{U}_{\alpha}$ one has

$$
\left.\boldsymbol{h}\left(s, s^{\prime}\right)\right|_{u_{\alpha}}=\frac{1}{k^{2}} h\left(e_{\alpha, i},\left(\left(s \otimes e^{\alpha, i}\right)^{*} \star_{\text {Wick }}^{\prime}\left(s^{\prime} \otimes e^{\alpha, j}\right)\right) e_{\alpha, j}\right) .
$$

Proof: Again the proof is easily done using Proposition 4 , Lemma 6 and the Wick type property of $\bullet_{\text {Wick }}^{\prime}$.

Remark 5 Again the above expression globally defines the metric $\boldsymbol{h}$ and here $\boldsymbol{h}$ turns out to be canonical in the sense that given a deformation of the metric $h$ that satisfies (64) and (63) it coincides with $\boldsymbol{h}$.

\section{Morita equivalence of Wick and anti Wick products}

In this section we fix a series of closed two-forms $\Omega$ of type $(1,1)$. Then consider the Wick and anti Wick star product constructed out of $\Omega$ as in Theorem 1. Their characteristic classes satisfy

$$
c\left(\star_{\text {Wick }}\right)-c\left(\star_{\text {Wick }}\right)=-\left[R^{L_{\mathrm{can}}}\right]=2 \pi \mathrm{i} c_{1}\left(L_{\mathrm{can}}\right),
$$

whence $\star_{\text {Wick }}$ and $\star_{\text {Wick }}$ are known to be Morita equivalent [9, Thm. 3.1]. Moreover, as the difference of their characteristic classes is given by the Chern class of $L_{\text {can }}$ a Morita equivalence bimodule is obtained by deforming $\Gamma^{\infty}\left(L_{\text {can }}\right)$ into a bimodule for $\star_{\text {Wick }}$ from the left and $\star_{\overline{\text { Wick }}}$ from the right. Finally, such a bimodule deformation necessarily exists. However, the concrete bimodule structure usually depends on non-canonical choices, even within its equivalence class of bimodule deformations, see e.g. the constructions in [8, 9] as well as the discussion in [7]. Thus one may ask the question whether in our particular situation there is a canonical construction, i.e. only using the Kähler geometry, of a Morita equivalence bimodule structure for $L_{\text {can }}$. As we shall show now this is indeed the case. 
First we consider the space $\mathcal{W} \otimes \Lambda^{\bullet} \otimes \mathcal{L}$ where $\mathcal{L}=\Gamma^{\infty}\left(L_{\text {can }}\right)$. We equip $\mathcal{W} \otimes \Lambda^{\bullet}$ with the fibrewise products $\circ_{\text {Wick }}$ and $\circ_{\text {Wick }}$ as before. But for $\mathcal{W} \otimes \Lambda^{\bullet} \otimes \mathcal{L}$ we use different bimodule multiplications, namely

$$
a \diamond_{\text {Wick }} \Psi=S^{-1} a \circ_{\text {Weyl }} \Psi \quad \text { and } \quad \Psi \diamond_{\overline{\text { Wick }}} b=\Psi \circ_{\text {Weyl }} S b,
$$

for $a, b \in \mathcal{W} \otimes \Lambda^{\bullet}$ and $\Psi \in \mathcal{W} \otimes \Lambda^{\bullet} \otimes \mathcal{L}$. Then we indeed obtain a bimodule as a simple computation shows:

Lemma 7 Using $\diamond_{\text {Wick }}$ and $\diamond_{\text {Wick }}$ the space $\mathcal{W} \otimes \Lambda^{\bullet} \otimes \mathcal{L}$ becomes a bimodule for $\left(\mathcal{W} \otimes \Lambda^{\bullet}, \circ_{\text {Wick }}\right)$ from the left and for $\left(\mathcal{W} \otimes \Lambda^{\bullet}, \circ \overline{\text { Wick }}\right)$ from the right.

Now let $r_{\text {Wick }}$ and $r_{\overline{\text { Wick }}}$ be the curvature elements as in Theorem 1, where we assume to have the same $\Omega$. Moreover, as connection $\nabla^{L_{\text {can }}}$ we use the canonical connection induced by the Kähler connection, see (80). Then we define $\mathcal{D}^{L}: \mathcal{W} \otimes \Lambda^{\bullet} \otimes \mathcal{L} \rightarrow \mathcal{W} \otimes \Lambda^{\bullet+1} \otimes \mathcal{L}$ explicitly by

$$
\mathcal{D}^{L} \Psi:=-\delta \Psi+D^{L} \Psi+\frac{\mathrm{i}}{\lambda}\left(r_{\text {Wick }} \diamond_{\text {Wick }} \Psi-(-1)^{\operatorname{deg}_{\mathrm{a}} \Psi} \Psi \diamond_{\overline{\text { Wick }}} r_{\overline{\text { Wick }}}\right) .
$$

Since the lowest orders of $r_{\text {Wick }}$ and $r_{\overline{\text { Wick }}}$ coincide the map $\mathcal{D}^{L}$ is well-defined, i.e. it does not produce negative powers of $\lambda$.

Lemma 8 The map $\mathcal{D}^{L}$ is a module derivation along $\mathcal{D}_{\text {Wick }}$ and $\mathcal{D}_{\overline{\text { Wick }}}$, respectively, i.e.

$$
\begin{aligned}
& \mathcal{D}^{L}\left(a \diamond_{\text {Wick }} \Psi\right)=\mathcal{D}_{\text {Wick }} a \diamond_{\text {Wick }} \Psi+(-1)^{\operatorname{deg}_{\mathrm{a}} a} a \diamond_{\text {Wick }} \mathcal{D}^{L} \Psi, \\
& \mathcal{D}^{L}\left(\Psi \diamond_{\overline{\text { Wick }}} b\right)=\mathcal{D}^{L} \Psi \diamond_{\text {Wick }} b+(-1)^{\operatorname{deg}_{\mathrm{a}} \Psi} \Psi \diamond_{\text {Wick }} \mathcal{D}_{\overline{\text { Wick }}} b .
\end{aligned}
$$

Proof: This is a straightforward computation using the properties of $\delta, D^{L}$, and $S$.

Lemma 9 For the symplectic curvature $R$ of the Kähler connection we have

$$
\begin{gathered}
R \diamond_{\text {Weyl }} \Psi=R \diamond_{\text {Wick }} \Psi+\lambda \varrho \Psi \quad \text { and } \quad \Psi \diamond_{\text {Weyl }} R=\Psi \diamond_{\overline{\text { Wick }}} R-\lambda \varrho \Psi, \\
\left(D^{L}\right)^{2}=\frac{\mathrm{i}}{\lambda}\left(R \diamond_{\text {Wick }} \Psi-\Psi \diamond_{\overline{\text { Wick }}} R\right) .
\end{gathered}
$$

Proof: The equation (72) follows directly from the definitions and $S R=R+\lambda \varrho$. Moreover, (82) together with Lemma 11 applied for $E=L_{\text {can }}$ and (72) implies (73).

Theorem 4 We have $\left(\mathcal{D}^{L}\right)^{2}=0$ and

$$
\sigma: \operatorname{ker} \mathcal{D}^{L} \cap \mathcal{W} \otimes \Lambda^{0} \otimes \mathcal{L} \rightarrow \Gamma^{\infty}\left(L_{\text {can }}\right)[[\lambda]]
$$

is a $\mathbb{C}[[\lambda]]$-linear bijection with inverse denoted by $\tau^{L_{\mathrm{can}}}$. Hence

$$
\begin{aligned}
& f \diamond_{\text {Wick }} s=\sigma\left(\tau_{\text {Wick }}(f) \diamond_{\text {Wick }} \tau^{L_{\text {can }}}(s)\right) \\
& s \diamond_{\overline{\text { Wick }}} g=\sigma\left(\tau^{L_{\text {can }}}(s) \diamond_{\overline{\text { Wick }}} \tau_{\overline{\text { Wick }}}(g)\right)
\end{aligned}
$$

defines a bimodule structure on $\Gamma^{\infty}\left(L_{\mathrm{can}}\right)[[\lambda]]$ for $\star_{\text {Wick }}$ from the left and $\star_{\overline{\text { Wick }}}$ from the right, deforming the classical bimodule structure of $\Gamma^{\infty}\left(L_{\mathrm{can}}\right)$. 
Proof: To compute $\left(\mathcal{D}^{L}\right)^{2}=0$ one has to use Lemma 9 and the particular properties of $r_{\text {Wick }}$ and $r_{\overline{\text { Wick }}}$ as in Theorem 1. Taking into account that we have used the same $\Omega$ for $r_{\text {Wick }}$ and $r_{\overline{\text { Wick }}}$ it easily follows that $\left(\mathcal{D}^{L}\right)^{2}=0$. The fact that (74) is a bijection is proved in the usual fashion by examining the fixed point equation

$$
\tau^{L}(s)=s+\delta^{-1}\left(D^{L} \tau^{L}(s)+\frac{\mathrm{i}}{\lambda} r_{\text {Wick }} \diamond_{\text {Wick }} \tau^{L}(s)-\frac{\mathrm{i}}{\lambda} \tau^{L}(s) \diamond_{\overline{\text { Wick }}} r_{\overline{\text { Wick }}}\right)
$$

for $s \in \Gamma^{\infty}\left(L_{\text {can }}\right)[[\lambda]]$ of the strictly contracting operator defined by the right hand side, analogously to [35, Thm. 3]. Then (75) and (76) obviously define a bimodule structure deforming the classical one.

Corollary 3 The bimodule $\left(\Gamma^{\infty}\left(L_{\mathrm{can}}\right)[[\lambda]], \star_{\text {Wick }}, \overline{\text { Wick }}\right)$ is a Morita equivalence bimodule for $\star_{\text {Wick }}$ and $\star_{\text {Wick }}$.

Proof: This follows from the general argument in [35, Prop. 1].

Remark 6 Clearly, the above construction is canonical in so far as it uses only the Kähler geometry. Hence there exists a distinguished bimodule structure on $\Gamma^{\infty}\left(L_{\mathrm{can}}\right)[[\lambda]]$ as desired. Note however, that ${ }_{\text {Wick }}$ and $\downarrow_{\text {Wick }}$ do not have any separation of variables properties.

Remark 7 Surprisingly, the Weyl product for $\Omega$ is not Morita equivalent to $\star_{\text {Wick }}$ or $\star_{\overline{W i c k}}$ in general as their relative class is given by $c\left(\star_{\text {Weyl }}\right)-c\left(\star_{\text {Wick }}\right)=-\frac{1}{2}\left[R^{L_{\text {can }}}\right]=\pi \mathrm{i} c_{1}\left(L_{\text {can }}\right)$. Thus their relative class needs not to be $2 \pi \mathrm{i}$ integral in general.

\section{A Some Kähler geometry}

In this appendix we shall recall some basic structures on Kähler manifolds in order to set up our notation and specify our sign conventions, see e.g. [27,38] for details.

Let $(M, I, g, \omega)$ be a Kähler manifold where $I$ denotes the complex structure, $g$ the Kähler metric, and $\omega$ the symplectic Kähler form. Since $I$ is integrable we have holomorphic local coordinates around any point. If $z^{1}, \ldots, z^{n}$ are such holomorphic local coordinates on $\mathcal{U} \subseteq M$ then we use the abbreviation $Z_{k}=\frac{\partial}{\partial z^{k}}$ and $\bar{Z}_{\ell}=\frac{\partial}{\partial \bar{z}^{\ell}}$. Locally, the $Z_{k}$ span the eigenbundle of $I$ to eigenvalue $+\mathrm{i}$ while the $\bar{Z}_{\ell}$ span the eigenbundle of $I$ to eigenvalue $-\mathrm{i}$. In such holomorphic coordinates the Kähler metric and the Kähler form are given by

$$
\left.g\right|_{u}=\frac{1}{2} g_{k \bar{\ell}} d z^{k} \vee d \bar{z}^{\ell} \quad \text { and }\left.\quad \omega\right|_{u}=\frac{\mathrm{i}}{2} g_{k \bar{\ell}} d z^{k} \wedge d \bar{z}^{\ell}
$$

By $\bigwedge^{(p, q)} T^{*} M$ we denote the bundle of $p+q$ forms of type $(p, q)$. In particular,

$$
L_{\text {can }}:=\bigwedge^{(n, 0)} T^{*} M
$$

is the so-called canonical line bundle of 'holomorphic $n$-forms'. Locally, a section $s \in \Gamma^{\infty}\left(L_{\text {can }}\right)$ can be written as $\left.s\right|_{\mathcal{U}}=f d z^{1} \wedge \cdots \wedge d z^{n}$ with $f \in C^{\infty}(\mathcal{U})$. The Kähler connection $\nabla$ extends to a connection $\nabla^{L_{\text {can }}}$ for $L_{\text {can }}$ which locally is given by

$$
\nabla_{X}^{L_{\mathrm{can}}} s=\left(X(f)-d z^{k}(X) \Gamma_{k \ell}^{\ell} f\right) d z^{1} \wedge \cdots \wedge d z^{n} .
$$


Here $\Gamma_{k m}^{\ell}=d z^{\ell}\left(\nabla_{Z_{k}} Z_{m}\right)$ are the Christoffel symbols of the Kähler connection. As $L_{\text {can }}$ is a line bundle the curvature of $\nabla^{L_{\text {can }}}$ is just a two-form $R^{L_{\text {can }}} \in \Gamma^{\infty}\left(\bigwedge^{(1,1)} T^{*} M\right)$ which is of type $(1,1)$. Locally one has

$$
R^{L_{\mathrm{can}}}=\Gamma_{k j, \bar{\ell}}^{j} d z^{k} \wedge d \bar{z}^{\ell}=-R_{j k \bar{\ell}}^{j} d z^{k} \wedge d \bar{z}^{\ell}
$$

where $R_{m k \bar{\ell}}^{j}=d z^{j}\left(\hat{R}\left(Z_{k}, \bar{Z}_{\ell}\right) Z_{m}\right)$ are the components of the curvature tensor $\hat{R}$ of $\nabla$. The Ricci form $\varrho$ is defined by

$$
\varrho=\frac{\mathrm{i}}{2} R^{L_{\mathrm{can}}}=-\frac{\mathrm{i}}{2} R_{j k \bar{\ell}}^{j} d z^{k} \wedge d \bar{z}^{\ell}
$$

whence we obtain the following relations for the (first) Chern class $c_{1}\left(L_{\text {can }}\right)$ of the canonical line bundle

$$
c_{1}\left(L_{\mathrm{can}}\right)=\frac{\mathrm{i}}{2 \pi}\left[R^{L_{\mathrm{can}}}\right]=\frac{1}{\pi}[\varrho] \in \mathrm{H}_{\mathrm{deRham}}^{2}(M, \mathbb{Z}) .
$$

Note that $\frac{1}{\pi} \varrho$ is integral but $\frac{1}{2 \pi} \varrho$ does not need to be integral.

The symplectic curvature tensor $R \in \Gamma^{\infty}\left(\bigvee^{(1,1)} T^{*} M \otimes \bigwedge^{(1,1)} T^{*} M\right)$ is defined as usual by

$$
R(X, Y, Z, W)=\omega(X, \hat{R}(Z, W) Y)
$$

for $X, Y, Z, W \in \Gamma^{\infty}(T M)$ and locally one has

$$
R=\frac{\mathrm{i}}{2} g_{k \bar{m}} R_{\bar{\ell} i \bar{j}}^{\bar{m}} d z^{k} \vee d \bar{z}^{\ell} \otimes d z^{i} \wedge d \bar{z}^{j}
$$

The fact that the Kähler connection is metric implies $g_{k \bar{m}} R_{\bar{\ell} i \bar{j}}^{\bar{m}}=-g_{m \bar{\ell}} R_{k i \bar{j}}^{m}$ whence one easily obtains

$$
\Delta_{\mathrm{fib}} R=\varrho .
$$

We note that $\bar{R}=R$ and $\bar{\varrho}=\varrho$ are real tensor fields while $\overline{R^{L_{\mathrm{can}}}}=-R^{L_{\mathrm{can}}}$ is imaginary.

Now let $E \rightarrow M$ be a holomorphic vector bundle of fibre dimension $k$ with Hermitian fibre metric $h$. By $e=\left(e_{1}, \ldots, e_{k}\right)$ we denote a local holomorphic frame of $E$, i.e. $e_{i} \in \Gamma^{\infty}\left(\left.E\right|_{u}\right)$ are holomorphic local base sections of $E$. Any local section $s \in \Gamma^{\infty}\left(\left.E\right|_{\mathcal{U}}\right)$ can be written as $s=e_{i} s^{i}$ with unique local smooth functions $s^{i} \in C^{\infty}(\mathcal{U})$. Then $s$ is holomorphic if and only if the $s^{i}$ are holomorphic. Different local holomorphic frames $e_{\alpha}$ and $e_{\beta}$ on $\mathcal{U}_{\alpha}$ and $\mathfrak{U}_{\beta}$, respectively, give rise to holomorphic transition matrices $\phi_{\alpha \beta}=\phi_{\beta \alpha}^{-1} \in M_{k}\left(C^{\infty}\left(\mathcal{U}_{\alpha} \cap \mathcal{U}_{\beta}\right)\right)$ by $e_{\beta}=e_{\alpha} \phi_{\alpha \beta}$. As usual they satisfy the co-cycle identity

$$
\phi_{\alpha \beta} \phi_{\beta \gamma} \phi_{\gamma \alpha}=\mathrm{id}
$$

on triple overlaps $\mathfrak{U}_{\alpha} \cap \mathcal{U}_{\beta} \cap \mathcal{U}_{\gamma}$. The coefficients of a section $s$ transform according to $s_{\alpha}=\phi_{\alpha \beta} s_{\beta}$.

A connection $\nabla^{E}$ for $E$ gives rise to a matrix $A$ of local connection one-forms $A \in M_{k}\left(\Gamma^{\infty}\left(T^{*} \mathfrak{U}\right)\right)$ with respect to a local holomorphic frame $e$ by

$$
\nabla_{X}^{E} e=-\mathrm{i} e A(X) \text { for } \quad X \in \Gamma^{\infty}(T M)
$$

Then $\nabla^{E}$ is called compatible with the holomorphic structure if $\nabla_{X}^{E} s=0$ for all locally holomorphic sections $s$ and all vector fields $X$ of type $(0,1)$. Equivalently, the connection one-forms $A$ with 
respect to any holomorphic frame are of type $(1,0)$. The connection is called compatible with the Hermitian fibre metric if

$$
X\left(h\left(s, s^{\prime}\right)\right)=h\left(\nabla \frac{E}{X} s, s^{\prime}\right)+h\left(s, \nabla_{X}^{E} s^{\prime}\right)
$$

for all $X \in \Gamma^{\infty}(T M)$ and $s, s^{\prime} \in \Gamma^{\infty}(E)$. This gives the local condition $d H=\mathrm{i}\left(A^{*} H-H A\right)$, where $H \in M_{k}\left(C^{\infty}(\mathcal{U})\right)$ is the local Hermitian matrix $H_{i j}=h\left(e_{i}, e_{j}\right)$ defined by the frame $e$. It is well-known that there exists a unique connection $\nabla^{E}$ which is compatible with both structures, the holomorphic structure and the Hermitian fibre metric, see e.g. [38, Chap. III, Sec. 2]. We shall refer to this connection as the canonical connection of $(E, h)$. Locally in a holomorphic frame one has

$$
A=\mathrm{i} H^{-1} \partial H
$$

Moreover, the curvature tensor $R^{E} \in \Gamma^{\infty}\left(\bigwedge^{2} T^{*} M \otimes \operatorname{End}(E)\right)$ is of type $(1,1)$. Finally, analogous statements hold for anti-holomorphic vector bundles as well.

\section{References}

[1] Bayen, F., Flato, M., Frønsdal, C., Lichnerowicz, A., Sternheimer, D.: Deformation Theory and Quantization. Ann. Phys. 111 (1978), 61-151.

[2] Berezin, F. A.: General Concept of Quantization. Commun. Math. Phys. 40 (1975), 153-174.

[3] Berezin, F. A.: Quantization. Math. USSR Izvestija 8.5 (1975), 1109-1165.

[4] Bertelson, M., Cahen, M., Gutt, S.: Equivalence of Star Products. Class. Quantum Grav. 14 (1997), A93-A107.

[5] Bordemann, M., Neumaier, N., Waldmann, S.: Homogeneous Fedosov Star Products on Cotangent Bundles I: Weyl and Standard Ordering with Differential Operator Representation. Commun. Math. Phys. 198 (1998), 363-396.

[6] Bordemann, M., Waldmann, S.: A Fedosov Star Product of Wick Type for Kähler Manifolds. Lett. Math. Phys. 41 (1997), 243-253.

[7] Bursztyn, H., Waldmann, S.: Bimodule deformations, Picard groups and contravariant connections. In preparation.

[8] Bursztyn, H., Waldmann, S.: Deformation Quantization of Hermitian Vector Bundles. Lett. Math. Phys. 53 (2000), 349-365.

[9] Bursztyn, H., Waldmann, S.: The characteristic classes of Morita equivalent star products on symplectic manifolds. Commun. Math. Phys. 228 (2002), 103-121.

[10] Cahen, M., Gutt, S., Rawnsley, J.: Quantization of Kähler Manifolds I: Geometric Interpretation of Berezin's Quantization. J. Geom. Phys. 7 (1990), 45-62.

[11] Cahen, M., Gutt, S., Rawnsley, J.: Quantization of Kähler Manifolds. II. Trans. Am. Math. Soc. 337.1 (1993), 73-98.

[12] Cahen, M., Gutt, S., Rawnsley, J.: Quantization of Kähler Manifolds. III. Lett. Math. Phys. 30 (1994), 291-305.

[13] Cahen, M., Gutt, S., Rawnsley, J.: Quantization of Kähler Manifolds. IV. Lett. Math. Phys. 34 (1995), $159-168$.

[14] DeWilde, M., Lecomte, P. B. A.: Existence of Star-Products and of Formal Deformations of the Poisson Lie Algebra of Arbitrary Symplectic Manifolds. Lett. Math. Phys. 7 (1983), 487-496.

[15] Dito, G., Sternheimer, D.: Deformation quantization: genesis, developments and metamorphoses. In: Halbout, G. (EDS.): Deformation quantization. [20], 9-54.

[16] Fedosov, B. V.: A Simple Geometrical Construction of Deformation Quantization. J. Diff. Geom. 40 (1994), 213-238.

[17] Fedosov, B. V.: Deformation Quantization and Index Theory. Akademie Verlag, Berlin, 1996. 
[18] Gutt, S.: Variations on deformation quantization. In: Dito, G., Sternheimer, D. (EDS.): Confèrence Moshè Flato 1999. Quantization, Deformations, and Symmetries, Mathematical Physics Studies no. 21, 217254. Kluwer Academic Publishers, Dordrecht, Boston, London, 2000.

[19] Gutt, S., Rawnsley, J.: Equivalence of star products on a symplectic manifold; an introduction to Deligne's Čech cohomology classes. J. Geom. Phys. 29 (1999), 347-392.

[20] Halbout, G. (EDs.): Deformation Quantization, vol. 1 in IRMA Lectures in Mathematics and Theoretical Physics. Walter de Gruyter, Berlin, New York, 2002.

[21] Hawkins, E.: Geometric Quantization of Vector Bundles and the Correspondence with Deformation Quantization. Commun. Math. Phys. 215 (2000), 409-432.

[22] Karabegov, A. V.: Deformation Quantization with Separation of Variables on a Kähler Manifold. Commun. Math. Phys. 180 (1996), 745-755.

[23] Karabegov, A. V.: Cohomological Classification of Deformation Quantizations with Separation of Variables. Lett. Math. Phys. 43 (1998), 347-357.

[24] Karabegov, A. V.: On Fedosov's approach to Deformation Quantization with Separation of Variables. In: Dito, G., Sternheimer, D. (EDS.): Confèrence Moshè Flato 1999. Quantization, Deformations, and Symmetries, Mathematical Physics Studies no. 22. Kluwer Academic Publishers, Dordrecht, Boston, London, 2000.

[25] Karabegov, A. V., Schlichenmaier, M.: Almost-Kähler Deformation Quantization. Lett. Math. Phys. 57 (2001), 135-148.

[26] Karabegov, A. V., Schlichenmaier, M.: Identification of Berezin-Toeplitz deformation quantization. J. reine angew. Math. 540 (2001), 49-76.

[27] Kobayashi, S., Nomizu, K.: Foundations of Differential Geometry II. Interscience Tracts in Pure and Applied Mathematics no. 15. John Wiley \& Sons, New York, London, Sydney, 1969.

[28] Kontsevich, M.: Deformation Quantization of Poisson Manifolds, I. Preprint q-alg/9709040 (September 1997).

[29] Landsman, N. P.: Mathematical Topics between Classical and Quantum Mechanics. Springer Monographs in Mathematics. Springer-Verlag, Berlin, Heidelberg, New York, 1998.

[30] Nest, R., Tsygan, B.: Algebraic Index Theorem. Commun. Math. Phys. 172 (1995), 223-262.

[31] Neumaier, N.: Local $\nu$-Euler Derivations and Deligne's Characteristic Class of Fedosov Star Products and Star Products of Special Type. Preprint Freiburg FR-THEP-99/3, math.QA/9905176 (June 1999). To appear in Commun. Math. Phys.

[32] Neumaier, N.: Universality of Fedosov's Construction for Star Products of Wick type on Semi-Kähler manifolds. Preprint Freiburg FR-THEP-2002/07 math.QA/0204031 (April 2002).

[33] Omori, H., Maeda, Y., Yoshioka, A.: Weyl Manifolds and Deformation Quantization. Adv. Math. 85 (1991), 224-255.

[34] Schlichenmaier, M.: Deformation Quantization of Compact Kähler Manifolds via Berezin-Toeplitz Operators. In: Doebner, H.-D., Nattermann, P., Scherer, W. (eds.): Group 21. Physical Applications and Mathematical Aspects of Geometry, Groups, and Algebras, 396-400. World Scientific, Singapore, New Jersey, London, Hong Kong, 1997. Proceedings of the XXI International Colloquium on Group Theoretical Methods in Physics.

[35] Waldmann, S.: Morita equivalence of Fedosov star products and deformed Hermitian vector bundles. Lett. Math. Phys. 60 (2002), 157-170.

[36] Waldmann, S.: On the representation theory of deformation quantization. In: Halbout, G. (EDS.): Deformation quantization. [20], 107-133.

[37] Weinstein, A., XU, P.: Hochschild cohomology and characteristic classes for star-products. In: KHOVANSKIJ, A., Varchenko, A., Vassiliev, V. (EDs.): Geometry of differential equations. Dedicated to V. I. Arnold on the occasion of his 60th birthday, 177-194. American Mathematical Society, Providence, 1998.

[38] Wells, R. O.: Differential Analysis on Complex Manifolds, vol. 65 in Graduate Texts in Mathematics. SpringerVerlag, New York, Berlin, Heidelberg, 1980. 